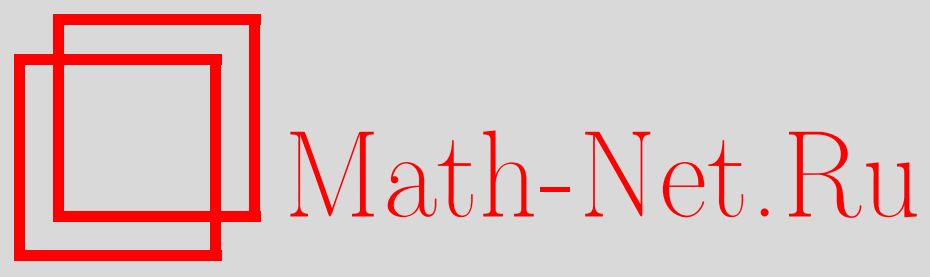

В. З. Гринес, Е. В. Жужома, В. С. Медведев, О диффеоморфизмах Морса-Смейла с четырьмя периодическими точками на замкнутых ориентируемых многообразиях, Матем. заметки, 2003, том 74, выпуск 3, 369-386

DOI: https://doi.org/10.4213/mzm271

Использование Общероссийского математического портала Math-Net.Ru подразумевает, что вы прочитали и согласны с пользовательским соглашением http://www . mathnet.ru/rus/agreement

Параметры загрузки:

IP : 3.82 .47 .9

26 апреля 2023 г., 13:24:51

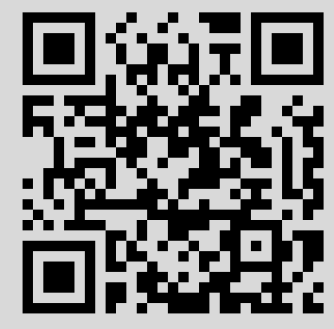




\title{
О ДИФФЕОМОРФИЗМАХ МОРСА-СМЕЙЛА С ЧЕТЫРЬМЯ ПЕРИОДИЧЕСКИМИ ТОЧКАМИ НА ЗАМКНУТЫХ ОРИЕНТИРУЕМЫХ МНОГООБРАЗИЯХ
}

\author{
В.З. Гринес, Е. В. Жужома, В. С. Медведев
}

\begin{abstract}
В статье рассматриваются диффеоморфизмы Морса-Смейла с четырьмя периодическими точками, которые исчерпьвают все множество периодических точек. Для $n=3$ доказывается градиентноподобность, и выделяется класс диффеоморфизмов, необходимо имеющих незамкнутую гетероклиническую кривую. Для $n \geqslant 4$ в классе диффеоморфизмов с одним седлом коразмерности один строится полный инвариант сопряженности.
\end{abstract}

Бибилиограффия: 15 названий.

1. Введение. Известно, что простейшими со многих точек зрения (нулевая энтропия, конечное множество неблуждающих точек и т.д.) структурно устойчивыми диффеоморфизмами являются диффеоморфизмы Морса-Смейла. Самым простьм из них является сохраняющий ориентацию диффеоморфизм замкнутого многообразия с двумя неподвижными точками, одна из которых есть тривиальный репеллер (источник), а другая - тривиальный аттрактор (сток), и других периодических точек нет ${ }^{1}$. В этом случае многообразие является сферой, и динамика такого диффеоморфизма проста: все орбиты, отличные от неподвижных точек, движутся от источника к стоку. Любые такие диффеоморфизмы на сфере фиксированной размерности топологически сопряжены.

Элементарные соображения показьвают, что на замкнутых ориентируемых двумерных многообразиях не существует диффеоморфизмов Морса-Смейла ровно с тремя периодическими точками. Можно показать, что на ориентируемых замкнутых трехмерных многообразиях также не существует диффеоморфизмов Морса-Смейла, неблуждающее множество которых состоит в точности из трех периодических точек (см. лемму 2.1). Поэтому естественно рассмотреть (с точки зрения проблемы топологической сопряженности, а также с точки зрения связи между динамическими свойствами и топологией несущего многообразия) диффеоморфизмы Морса-Смейла замкнутого ориентируемого $n$-мерного многообразия $M^{n}$, неблуждающие множества которых состоят в точности из четырех периодических точек.

Работа выполнена при поддержке Российского фонда фундаментальных исследований, грант № 02-01-000-98.

${ }^{1}$ Заметим, что на любом замкнутом многообразии диффеоморфизм Морса-Смейла имеет, по крайней мере, одну источниковую и одну стоковую периодические точки [1]. 
Для размерности $n=2$ такие диффеоморфизмы сушествуют только на двумерной сфере и их топологическая классификация следует из работ [2], [3]. Поэтому в настоящей статье мы рассматриваем ориентируемые многообразия $M^{n}$ размерности $n \geqslant 3$. Вначале для $n=3$ мы доказываем следующий результат.

ТЕорема 1. Любой диффеоморфизм $f: M^{3} \rightarrow M^{3}$ Морса-Смейла, неблуждающее множество которого состоит в точности из четырех периодических точек, является градиентноподобным.

Перейдя к некоторой итерации диффеоморфизма Морса-Смейла, можно добиться того, чтобы его неблуждающее множество состояло только из неподвижных точек. Аналогичным образом получается диффеоморфизм, ограничение которого на инвариантное многообразие любой его неподвижной точки сохраняет ориентацию (данного инвариантного многообразия). Класс таких диффеоморфизмов Морса-Смейла с четырьмя неподвижными точками обозначим через $M S\left(M^{n}, 4\right)$. Так как любой диффеоморфизм из $M S\left(M^{n}, 4\right)$ содержит, по крайней мере, один источник и один сток, и не может иметь два источника и два стока одновременно, то множество $M S\left(M^{3}, 4\right)$ можно представить в виде объединения двух подклассов $M S_{1}\left(M^{3}, 4\right), M S_{2}\left(M^{3}, 4\right)$, соответствующих следующим наборам неподвижных точек:

1) источник, сток и два седла;

2) источник, два стока и седло, либо сток, два источника и седло.

Ниже для определенности мы будем считать, что $f$ из подкласса $M S_{2}\left(M^{3}, 4\right)$ содержит источник, два стока и седло (в противном случае можно перейти к обратному $f^{-1}$ ).

Диффеоморфизмы из $M S_{2}\left(M^{3}, 4\right)$ (на рис. 1(а) указан пример фазового портрета такого диффеоморфизма) могут сушествовать только на трехмерной сфере $S^{3}=M^{3}$. Диффеоморфизмы из $M S_{1}\left(M^{3}, 4\right)$ существуют также на линзах, поскольку линзы можно получить склеиванием двух полноторий. Мы показываем, что седла произвольного $f \in$ $M S_{1}\left(M^{3}, 4\right)$ имеют разные индексы Морса (лемма 2.2). Остается открытым вопрос, имеются ли замкнутые ориентируемые многообразия, отличные от сферы, $S^{2} \times S^{1}$ и линз, допускающие диффеоморфизмы из $M S_{1}\left(M^{3}, 4\right)$.

Несмотря на градиентноподобность и "малое" количество неподвижных точек, диффеоморфизмы из $M S\left(M^{3}, 4\right)$ могут демонстрировать достаточно сложное вложение инвариантных многообразий седловых периодических точек, что приводит к существованию счетного множества классов топологической сопряженности даже в классе $M S_{2}\left(S^{3}, 4\right)$. Этот класс $M S_{2}\left(S^{3}, 4\right)$ рассматривался в [4]-[6]. В этих работах была обнаружена возможность дикого вложения в $S^{3}$ одномерных и двумерных сепаратрис седловых неподвижных точек диффеоморфизмов из класса $M S_{2}\left(S^{3}, 4\right)$, и получена топологическая классификация таких диффеоморфизмов, которая свелась к классификации узлов в $S^{2} \times S^{1}$. Чтобы понять возникновение дикого вложения сепаратрис седловой неподвижной точки диффеоморфизма $f \in M S_{2}\left(S^{3}, 4\right)$, обозначим через $\omega_{1}, \omega_{2}$ стоковые точки и через $W^{u+}(\sigma)$ сепаратрису седловой точки $\sigma$, идущую в стоковую точку $\omega_{2}$ (см. рис. 1(a)). Теперь представим объединение $\alpha \cup W^{s}(\sigma) \cup W^{u+}(\sigma) \cup \omega_{2}$ как бесконечную “кривую", половина которой немного раздута (эта половина соответствует $\alpha \cup W^{s}(\sigma)$ ), где $W^{s}(\sigma)$ - устойчивое многообразие седловой неподвижной точки $\sigma$. Затем вложим эту “кривую" вместе с некоторой окрестностью в $S^{3}$ как кривую с двумя концами дикости, например, как хорошо известную дикую дугу Артина-Фокса [7] (см. рис. 1(b)). Построение соответствующих примеров на трехмерной сфере имеются в [4], [5]. 


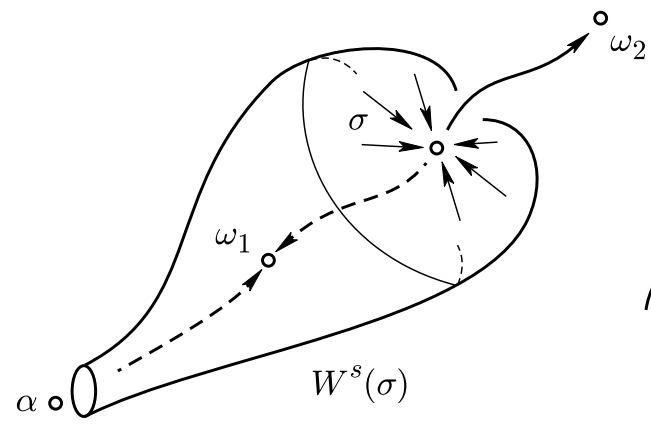

(a)

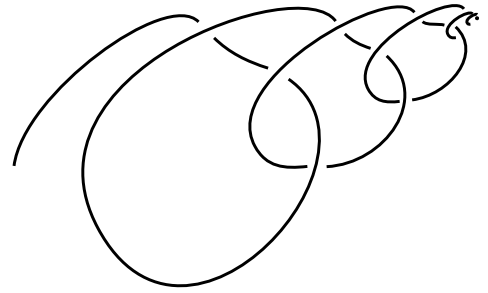

(b)

Рис. 1. Диффеоморфизм Морса-Смейла из класса $M S_{2}\left(M^{3}, 4\right)$.

Так как подкласс $M S_{2}\left(M^{3}, 4\right)$ расклассифицирован в работах [5], [6], мы более подробно рассматриваем подкласс $M S_{1}\left(M^{3}, 4\right)$, когда многообразие $M^{3}$ накрывается сферой $S^{3}$, и доказьваем, что в этом случае блуждающее множество любого диффеоморфизма из $M S_{1}\left(M^{3}, 4\right)$ содержит хотя бы одну незамкнутую гетероклиническую кривую.

Теорема 2. Пусть $f$-диффеоморфизм Морса-Смейла замкнутого ориентируемого трехмерного многообразия, для которого 3-мерная сфера $S^{3}$ является универсальным накрытием, и пусть неблуждающее множество $f$ состоит из двух седловых и двух узловых периодических точек. Тогда существует, по крайней мере, одна гетероклиническая незмкнутая кривая, граница которой состоит из седловых точек. Каждая такая гетероклиническая кривая инвариантна относительно некоторой итерации диффеоморфизма $f$.

Более того, если пересечение двумерных инвариантных многообразий седловых точек не исчерпывается такими гетероклиническими кривыми, то оставшаяся часть пересечения содержит счетное семейство замкнутых гетероклинических кривых, которые представляют собой оббединение орбит некоторого конечного набора замкнутых гетероклинических кривых.

На рис. 2 приводится фазовый портрет диффеоморфизма из $M S_{1}\left(S^{3}, 4\right)$, показьвающий как можно реализовать все возможности, описанные в теореме 2 . Более того, объединение $\alpha \cup W^{s}\left(\sigma_{1}\right) \cup W^{u}\left(\sigma_{2}\right) \cup \omega$ сепаратрис седел и узловых точек можно вложить вместе с некоторой окрестностью в $S^{3}$ как кривую с двумя концами дикости (например, как дикую дугу Артина-Фокса [7]; см. рис. 1(b)) аналогично конструкции в [5]. В результате мы получим градиентноподобный диффеоморфизм Морса-Смейла более сложный, чем в [5], поскольку полньй инвариант сопряженности такого диффеоморфизма, помимо "узловой" информации (достаточной для диффеоморфизмов из $M S_{2}(4)$ ), должен содержать информацию о структуре множества гетероклинических кривых.

Для размерностей $n \geqslant 4$ мы основное внимание уделяем подклассу диффеоморфизмов $M S_{n-1}\left(M^{n}, 4\right) \subset M S\left(M^{n}, 4\right)$, неблуждающее множество которых состоит из трех узлов и одного седла коразмерности один. Очевидно, любой диффеоморфизм из $M S_{n-1}\left(M^{n}, 4\right)$ является градиентноподобным. Мы доказываем следующую теорему.

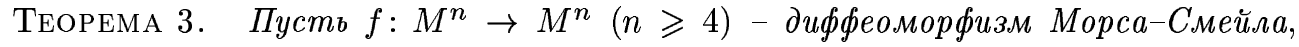




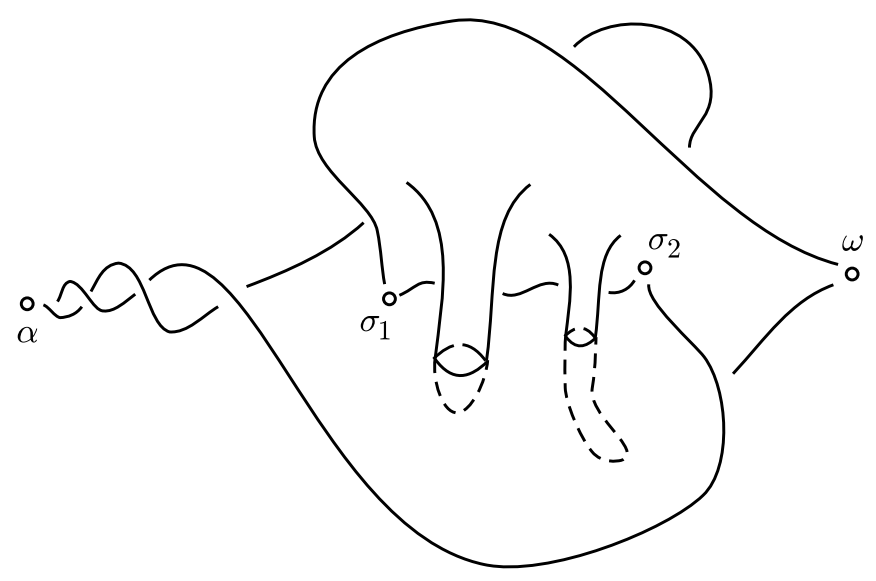

Рис. 2. Пример фазового портрета диффеоморфизма из $M S_{1}(4)$.

неблуждающее множество $N W(f)$ которого состоит из трех узловых периодических точек и одного седла б коразмерности один. Тогда

1) многообразие $M^{n}$ есть $n$-мерная сфера $S^{n}$;

2) неустойчивое многообразие коразмерности один седла $\sigma$ и один из узлов образуют иилиндрически вложенную $(n-1)$-мерную сферу, которая ограничивает в $S^{n}$ n-мерный шар, содержащий ровно один узел;

3) одномерные сепаратрисы седла содержст в своем замыкании различные узлы.

Следующая теорема решает проблему топологической классификации диффеоморфизмов из $M S_{n-1}\left(M^{n}, 4\right)$.

ТЕорема 4. Два диффеоморфизма $f, g \in M S_{n-1}\left(M^{n}, 4\right)$ сопряжсены тогда $и$ только тогда, когда их седла $\sigma_{f}, \sigma_{g}$ имеют одинаковый индекс Морса (либо 1 , либо $n-1)$.

Эта теорема контрастирует с ситуацией в трехмерном случае, где для фиксированного индекса Морса седла существует счетное множество попарно несопряженных градиентноподобных диффеоморфизмов Морса-Смейла [5]. Простота "многомерного" случая обусловлена леммой 3.1 , в которой показьвается, что одномерные сепаратрисы седел в силу “многомерности” тривиально заузлены.

Напомним некоторые понятия и факты, касающиеся диффеоморфизмов МорсаСмейла. Хорошим источником являются книга [8], а также обзорные статьи [1], [9] и $[10]$.

Множество неблуждающих точек диффеоморфизма $f$ будем обозначать через $N W(f)$. Для $x \in N W(f)$ обозначим через $W^{s}(x)$ (соответственно $W^{u}(x)$ ) устойчивое (соответственно неустойчивое) многообразие этой точки. Диффеоморфизм $f$ назьвает-

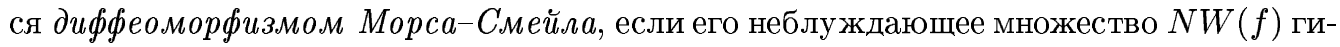
перболическое, состоит из конечного числа точек, и инвариантные многообразия $W^{s}(x)$, $W^{u}(y)$ пересекаются трансверсально (если пересечение не пусто) для любых точек $x, y \in N W(f)$. Диффеоморфизм $f$ Морса-Смейла назьвается градиентноподобным, 
если для любых периодических точек $p, q \in N W(f)$ из $W^{u}(p) \cap W^{s}(q) \neq \varnothing$ следует, что $\operatorname{dim} W^{s}(p)<\operatorname{dim} W^{s}(q)$.

Точка $x \in M$ трансверсального пересечения инвариантных многообразий $W^{s}(p)$, $W^{u}(q)$, где $p, q \in N W(f)$, назьвается гетероклинической, если $\operatorname{dim} W^{s}(p)=$ $\operatorname{dim} W^{s}(q)$. Диффеоморфизм Морса-Смейла является градиентноподобным диффеоморфизмом тогда и только тогда, когда он не имеет гетероклинических точек.

Если $W^{u}(p) \cap W^{s}(q) \neq \varnothing$ и $\operatorname{dim} W^{s}(p)<\operatorname{dim} W^{s}(q)$, то компоненту связности пересечения $W^{u}(p) \cap W^{s}(q)$ назовем гетероклиническим подмногообразием. Если размерность многообразия равна 3, то любое гетероклиническое подмногообразие является либо простой замкнутой кривой (гомеоморфной окружности), либо незамкнутой кривой без самопересечений (гомеоморфной открытому интервалу). Мы будем назьвать такие кривые гетероклиническими.

Пусть $p$ - периодическая точка диффеоморфизма Морса-Смейла $f$. Индексом Мор$c a$ точки $p$ называется топологическая размерность неустойчивого многообразия $W^{u}(p), u(p) \stackrel{\text { def }}{=} \operatorname{dim} W^{u}(p)$. Индексом Кронекера-Пуанкаре точки $p$ назьвается число $\operatorname{ind}(p, f) \stackrel{\text { def }}{=}(-1)^{u(p)}$.

Нам понадобятся некоторые понятия из теории узлов и из теории топологических вложений полиэдров и симплексов в многообразие. Напомним, что множество $P \subset M^{n}$, гомеоморфное стандартному полиэдру евклидова пространства, называется дико вложенным, если не существует гомеоморфного отображения $M^{n}$ на себя, при котором $P$ переходит в прямолинейньй полиэдр. В противном случае, $P$ называется $p y^{4}$ в $M^{n}[11]$.

2. Диффеоморфизмы $M S\left(M^{3}, 4\right)$. В этом разделе мы рассматриваем диффеоморфизмы Морса-Смейла замкнутых ориентируемых трехмерных многообразий $M^{3}$. Сначала покажем, что такие диффеоморфизмы не могут иметь ровно трех периодических точек. Для конечного множества $A$ обозначим через $|A|$ его мощность.

ЛЕмма 2.1. Пусть $f: M^{3} \rightarrow M^{3}-$ - дифеоморфизм Морса-Смейла. Тогда $|N W(f)| \neq 3$.

ДокАЗАТЕЛЬСтво. В самом деле, если такой диффеоморфизм $f$ существует, то его неблуждающее множество содержит в точности одно седло, один сток и один источник. Следовательно, $f$ не имеет гетероклинических кривых. В силу [12] для диффеоморфизма Морса-Смейла, не имеющего гетероклинических кривых, существует целое неотрицательное число $m$ такое, что имеет место формула $l-k=2-2 m$, где $l$ - число всех стоковых и источниковых периодических точек, и $k$ - число всех седловых периодических точек. Поэтому для $f$ должно вьполняться равенство $1=2-2 m$, которое невозможно ни при каком целом $m$.

Опишем набор неподвижных точек для диффеоморфизмов из класса $M S_{1}\left(M^{3}, 4\right)$.

Лемма 2.2. Если $f \in M S_{1}\left(M^{3}, 4\right)$, то $f$ имеет следующий набор неподвижных точек: источник $\alpha$, сток $\omega$ и две седловые точки $\sigma_{1}, \sigma_{2}$ с индексами Морса $u(\alpha)=3, u(\omega)=0, u\left(\sigma_{1}\right)=1, u\left(\sigma_{2}\right)=2$ соответственно.

ДокАЗАТЕЛьство. Достаточно показать, что седла $\sigma_{1}, \sigma_{2}$ имеют разньй индекс Морса. Предположим противное. Тогда $f$ не имеет гетероклинических кривых. В работе [12] доказано, что в этом случае $M^{3}$ есть связная сумма $m$ экземпляров $S^{2} \times S^{1}$. Так 
как $f \in M S_{1}\left(M^{3}, 4\right)$, то $m=1$ и $M^{3}=S^{2} \times S^{1}$. Применим теперь формулу Лефшеца

$$
L(f)=\sum_{p \in \text { Fix } f} \operatorname{ind}(p, f),
$$

где $L(f)$ - число Лефшеца, которое вычисляется по формуле

$$
L(f)=\sum_{i=0}^{n}(-1)^{i} \operatorname{Sp} f_{i *},
$$

$\operatorname{Sp} f_{i *}-$ след линейного отображения $f_{i *}: H_{i}\left(M^{3}, \mathbb{R}\right) \rightarrow H_{i}\left(M^{3}, \mathbb{R}\right) i$-мерной групшы гомологий $H_{i}\left(M^{3}, \mathbb{R}\right)$, индуцированного $f$. Для $M^{3}=S^{2} \times S^{1}$ и сохраняющего ориентацию $f$ имеем $L(f)=0$, поскольку

$$
H_{1}\left(M^{3}, \mathbb{R}\right)=H_{2}\left(M^{3}, \mathbb{R}\right)=\mathbb{Z}, \quad \operatorname{Sp} f_{1 *}=\operatorname{Sp} f_{2 *}=1
$$

С другой стороны в силу предположения о равенстве индексов Морса у седел сумма индексов неподвижных точек не может равняться нулю. Полученное противоречие доказывает лемму.

Везде далее в этом разделе для диффеоморфизма Морса-Смейла $f: M^{3} \rightarrow M^{3}$ из класса $M S\left(M^{3}, 4\right)$ будем использовать следующие обозначения его неподвижных точек:

1) если $f \in M S_{1}\left(M^{3}, 4\right)$, то $f$ имеет источник $\alpha$, сток $\omega$ и два седла $\sigma_{1}, \sigma_{2}$ с индексами Кронекера-Пуанкаре (соответственно Морса) ind $(\alpha, f)=-1(u(\alpha)=3)$, ind $(\omega, f)=1$ $(u(\omega)=0), \operatorname{ind}\left(\sigma_{1}, f\right)=-1\left(u\left(\sigma_{1}\right)=1\right), \operatorname{ind}\left(\sigma_{2}, f\right)=1\left(u\left(\sigma_{2}\right)=2\right)$;

2) если $f \in M S_{2}\left(M^{3}, 4\right)$, то $f$ имеет источник $\alpha$, два стока $\omega_{1}, \omega_{2}$ и седло $\sigma$ с индексами Кронекера-Пуанкаре (соответственно Морса) ind $(\alpha, f)=-1(u(\alpha)=3)$, $\operatorname{ind}\left(\omega_{1}, f\right)=1\left(u\left(\omega_{1}\right)=0\right)$, ind $\left(\omega_{2}, f\right)=1\left(u\left(\omega_{2}\right)=0\right), \operatorname{ind}(\sigma, f)=-1(u(\sigma)=1)$.

Лемма 2.3. Если $f \in M S_{1}\left(M^{3}, 4\right)$, то имеют место следующие включения:

$$
W^{u}\left(\sigma_{1}\right)-\sigma_{1} \subset W^{s}(\omega), \quad W^{s}\left(\sigma_{2}\right)-\sigma_{2} \subset W^{u}(\alpha) .
$$

ДокАЗАТЕЛЬСТво. Поскольку $f$ не может иметь гомоклинических точек, то $W^{s}\left(\sigma_{i}\right) \cap W^{u}\left(\sigma_{i}\right)=\varnothing(i=1,2)$. Так как $f$ - структурно устойчивьй диффеоморфизм, то $W^{u}\left(\sigma_{1}\right) \cap W^{s}\left(\sigma_{2}\right)=\varnothing$, иначе бы в точках пересечения не выполнялось сильное условие трансверсальности. Отсюда вытекают требуемые включения, так как $M^{3}$ разбивается на попарно не пересекающиеся инвариантные многообразия, устойчивые или неустойчивые соответственно.

Следующая лемма доказана в [5]. Мы приводим ее для ссылок, оставляя читателю доказательство в качестве упражнения.

Лемма 2.4. Eсли $f \in M S_{2}\left(M^{3}, 4\right), \operatorname{mo} W^{s}(\sigma)-\sigma \subset W^{u}(\alpha)$.

Покажем теперь, что диффеоморфизм $f: M^{3} \rightarrow M^{3}$ Морса-Смейла с четырьмя неподвижными точками не имеет гетероклинических точек. 
ДокАЗАТЕЛЬСТво ТЕоремы 1. Так как инвариантные множества периодических точек не меняются при переходе к итерации диффеоморфизма, не уменьшая общности, можно считать, что $f$ - сохраняющий ориентацию диффеоморфизм, ограничение которого на инвариантные многообразия неподвижных точек также сохраняет ориентацию, т.е. $f \in M S\left(M^{3}, 4\right)$. Возможны два случая: $f \in M S_{1}\left(M^{3}, 4\right)$ и $f \in M S_{2}\left(M^{3}, 4\right)$. В случае $f \in M S_{1}\left(M^{3}, 4\right)$ достаточно рассмотреть пересечения инвариантных многообразий седловых точек $\sigma_{1}, \sigma_{2}$ с индексами Морса $u\left(\sigma_{1}\right)=1, u\left(\sigma_{2}\right)=2$ соответственно. В силу леммы $2.3 W^{u}\left(\sigma_{1}\right)-\sigma_{1} \subset W^{s}(\omega)$ и $W^{s}\left(\sigma_{2}\right)-\sigma_{2} \subset W^{u}(\alpha)$. Следовательно, $W^{u}\left(\sigma_{1}\right)$ не пересекает $W^{s}\left(\sigma_{2}\right)$. Если же $W^{s}\left(\sigma_{1}\right) \cap W^{u}\left(\sigma_{2}\right) \neq \varnothing$, то выполняется неравенство $2=\operatorname{dim} W^{s}\left(\sigma_{1}\right)>\operatorname{dim} W^{u}\left(\sigma_{2}\right)=1$, что означает градиентноподобность.

В случае $f \in M S_{2}\left(M^{3}, 4\right)$ требуемьй результат вытекает из леммы 2.4, поскольку $f$ имеет ровно одну седловую точку.

Далее в этом разделе мы в основном рассматриваем диффеоморфизмы из класса $M S_{1}\left(M^{3}, 4\right)$, используя предыдущие обозначения для набора неподвижных точек.

Лемма 2.5. Пусть $f \in M S_{1}\left(M^{3}, 4\right)$. Тогда мнохества

$$
C_{\omega} \stackrel{\text { def }}{=}\{\omega\} \cup W^{u}\left(\sigma_{1}\right) \quad u \quad C_{\alpha} \stackrel{\text { def }}{=}\{\alpha\} \cup W^{s}\left(\sigma_{2}\right)
$$

являются вложсениями окружностей.

ДокАЗАТЕЛьСТво. Докажем лемму только для $C_{\omega}$, поскольку для $C_{\alpha}$ доказательство аналогичное.

Существует $C^{1}$-иммерсия $\varphi: \mathbb{R} \rightarrow W^{u}\left(\sigma_{1}\right)$, где $\varphi(0)=\sigma_{1}$, являющаяся взаимно однозначньм отображением на свой образ. Покажем, что иммерсия $\varphi$ может быть продолжена до гомеоморфизма

$$
\varphi: S^{1} \cong \mathbb{R} \cup\{\infty\} \rightarrow W^{u}\left(\sigma_{1}\right) \cup\left\{\sigma_{1}\right\}
$$

если положить $\varphi( \pm \infty)=\omega$. В силу леммы $2.3 W^{u}\left(\sigma_{1}\right)-\sigma_{1} \subset W^{s}(\omega)$. Тогда требуемое утверждение следует из того, что $\omega$-предельное множество любой точки из множества $W^{u}\left(\sigma_{1}\right)-\sigma_{1}$ есть точка $\omega$ (см. рис. 3$)$. Отсюда вытекает требуемое утверждение.
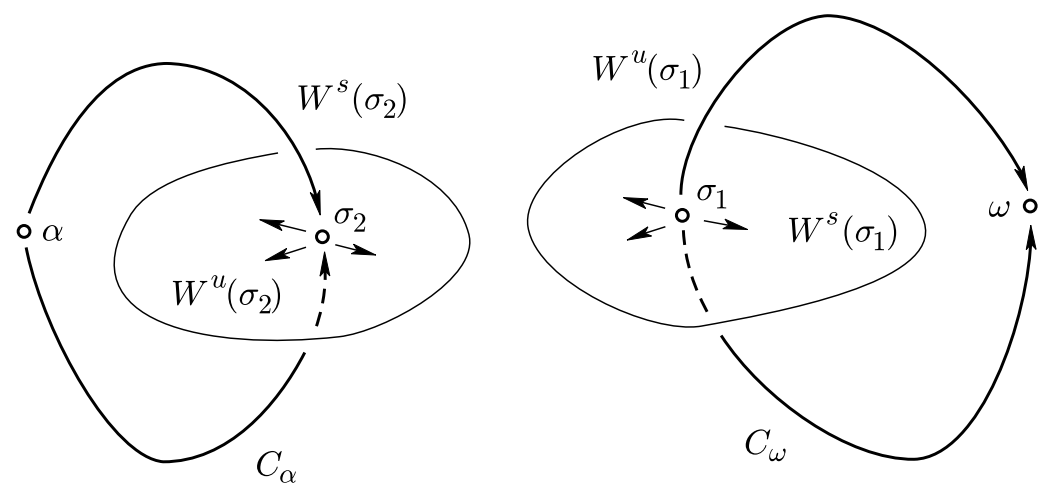

Рис. 3. Кривые $C_{\alpha}$ и $C_{\omega}$. 
ДокАЗАТЕЛЬСТво ТЕОРЕмы 2. Так как инвариантные множества периодических точек не меняются при переходе к итерации диффеоморфизма, не уменьшая общности, можно считать, что $f \in M S_{1}\left(M^{3}, 4\right)$. Сначала докажем, что $W^{s}\left(\sigma_{1}\right) \cap W^{u}\left(\sigma_{2}\right)$ содержит хотя бы одну незамкнутую гетероклиническую кривую с концевыми точками $\sigma_{1}$, $\sigma_{2}$. В устойчивом многообразии $W^{s}\left(\sigma_{1}\right)$ возьмем фундаментальную область $F^{s}$ диффеоморфизма $\left.f\right|_{W^{s}\left(\sigma_{1}\right)-\sigma_{1}}$. Так как точка $\sigma_{1}$ гиперболическая, можем считать $F^{s}$ замкнутым кольцом, ограниченным гладкими кривыми $C_{1}$ и $C_{2}$, которые окружают точку $\sigma_{1}$ в $W^{s}\left(\sigma_{1}\right)$. Возьмем в $F^{s}$ простую замкнутую кривую $C$, гомотопную $C_{1}$ и $C_{2}$. Для удобства разобьем дальнейшее доказательство на утверждения, которые мы будем обозначать как шаги.

ШАГ 2.1. Для любой замкнутой кривой $C$, гомотопной кривым $C_{1} u C_{2}$, пересечение $C \cap W^{u}\left(\sigma_{2}\right)$ непусто.

ДокАЗАТЕЛЬСТво ШАГА 2.1. ПредПоложим, что $C \cap W^{u}\left(\sigma_{2}\right)=\varnothing$. Тогда $C \subset$ $W^{u}(\alpha)$, поскольку $M^{3}-\omega$ есть объединение только трех попарно непересекающихся неустойчивьх многообразий $W^{u}(\alpha), W^{u}\left(\sigma_{2}\right)$ и $W^{u}\left(\sigma_{1}\right)$. В силу компактности $C_{\omega}=\{\omega\} \cup$ $W^{u}\left(\sigma_{1}\right)$ существует такая окрестность $U(\alpha)$ источника $\alpha$, что $U(\alpha) \cap C_{\omega}=\varnothing$. Из включения $C \subset W^{u}(\alpha)$ и компактности $C$ следует существование целого отрицательного числа $n_{0}$ такого, что $f^{n_{0}}(C) \subset U(\alpha)$.

Рассмотрим универсальное накрытие $q: S^{3} \rightarrow M^{3}$ и накрывающий диффеоморфизм $\tilde{f}: S^{3} \rightarrow S^{3}$. Пусть $\widetilde{C}_{\omega}-$ компонента множества $q^{-1}\left(C_{\omega}\right)$. Из конечнолистности накрытия $q$ вытекает, что $\widetilde{C}_{\omega}$ является простой замкнутой кривой, содержащей хотя бы одно седло $\tilde{\sigma}_{1} \in q^{-1}\left(\sigma_{1}\right)$. В силу $u\left(\sigma_{1}\right)=1 W^{s}\left(\sigma_{1}\right)$ есть образ $\mathbb{R}^{2}$ при инъективной иммерсии. Так как кривая $C \subset W^{s}\left(\sigma_{1}\right)-\sigma_{1}$ негомотопна нулю в $W^{s}\left(\sigma_{1}\right)-\sigma_{1}$, она ограничивает в $W^{s}\left(\sigma_{1}\right)$ диск $D$, содержащий точку $\sigma_{1}$. Поскольку $f$ не имеет гомоклинических точек, диск $D$ пересекается с $C_{\omega}$ ровно в одной точке $\sigma_{1}$. Поэтому на $W^{s}\left(\tilde{\sigma}_{1}\right)$ лежит простая замкнутая кривая $\widetilde{C} \subset q^{-1}(C)$, которая ограничивает на $W^{s}\left(\tilde{\sigma}_{1}\right)$ диск, пересекающийся с семейством замкнутых кривых из множества $q^{-1}\left(C_{\omega}\right)$ ровно в одной точке $\tilde{\sigma}_{1}$. Следовательно, $\widetilde{C}$ и $q^{-1}\left(C_{\omega}\right)$ образуют нетривиальное зацепление с коэффициентом зацепления -1 или +1 (в зависимости от ориентаций кривых). Тогда $\tilde{f}^{n_{0}}(\widetilde{C})$ и $\tilde{f}^{n_{0}}\left(q^{-1}\left(C_{\omega}\right)\right)$ также образуют нетривиальное зацепление с коэффициентом зацепления -1 или +1 . Из $f\left(C_{\omega}\right)=C_{\omega}$ вытекает равенство $\tilde{f}^{n_{0}}\left(q^{-1}\left(C_{\omega}\right)\right)=q^{-1}\left(C_{\omega}\right)$. Поэтому $\tilde{f}^{n_{0}}(\widetilde{C})$ и $q^{-1}\left(C_{\omega}\right)$ образуют нетривиальное зацепление.

С другой стороны, $\tilde{f}^{n_{0}}(\widetilde{C}) \subset q^{-1}(U(\alpha))$. Не уменьшая общности, можно считать, что множество $q^{-1}(U(\alpha))$ представляет собой конечное объединение попарно непересекающихся компонент, гомеоморфных $U(\alpha)$, и каждая компонента гомеоморфна трехмерному шару. Следовательно, на замкнутую кривую $\tilde{f}^{n_{0}}(\widetilde{C})$ можно натянуть поверхность $S$, полностью лежащую в одной из этих компонент и, следовательно, в $q^{-1}(U(\alpha))$. Так как $U(\alpha) \cap C_{\omega}=\varnothing$, отсюда получаем, что $S$ не пересекается с $q^{-1}\left(C_{\omega}\right)$. Следовательно, коэффищиент зацепления $\tilde{f}^{n_{0}}(\widetilde{C})$ и $q^{-1}\left(C_{\omega}\right)$ равен нулю. Мы получили противоречие.

Таким образом, $W^{s}\left(\sigma_{1}\right) \cap W^{u}\left(\sigma_{2}\right) \neq \varnothing$. Так как $W^{s}\left(\sigma_{1}\right)$ и $W^{u}\left(\sigma_{2}\right)$ пересекаются трансверсально, то пересечение $W^{s}\left(\sigma_{1}\right) \cap W^{u}\left(\sigma_{2}\right)$ состоит из кривых. В силу произвольности кривой $C$, пересечение $F^{s} \cap W^{u}\left(\sigma_{2}\right)$ содержит, по крайней мере, одну дугу $d$ с концевыми точками $a_{1}, a_{2}$, лежащими на разных граничных компонентах $C_{1}$ и $C_{2}$ кольца $F^{s}$. Для определенности положим $a_{i} \in C_{i}(i=1,2)$. Обозначим через $\mathcal{D}$ кривую из $W^{s}\left(\sigma_{1}\right) \cap W^{u}\left(\sigma_{2}\right)$, содержащую дугу $d$ (рис. 4$)$. 


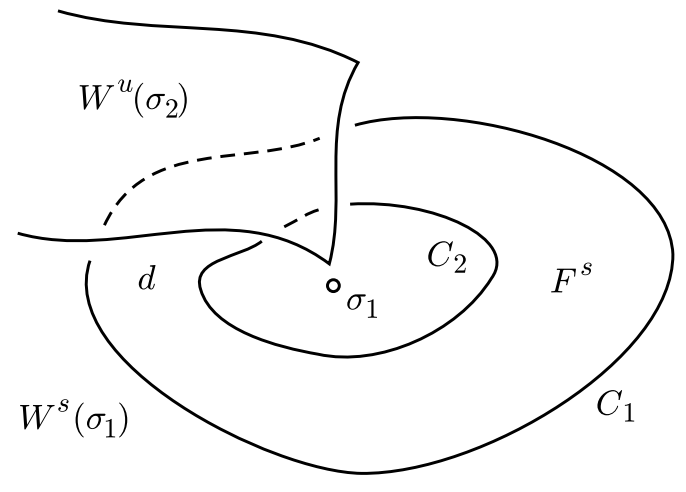

Рис. 4. Дуга $d$ в фундаментальном кольце $F^{s}$.

ШАГ 2.2. Для компактного (в топологии многообразия $W^{s}\left(\sigma_{1}\right)$ ) подмножества $F \subset W^{s}\left(\sigma_{1}\right)$ и любой точки $m_{0} \in \operatorname{int} F$ существует окрестность $U\left(m_{0}\right)$, которая гомеоморфна диску и которая пересекается не болеечем с одной кривой из пересечения $F \cap W^{u}\left(\sigma_{2}\right)$, при этом, если $U\left(m_{0}\right)$ пересекается с одной кривой, скажем $l$, то пересечение $U\left(m_{0}\right) \cap l$ состоит из одной компоненты, гомеоморфной простой дуге, которая делит $U\left(m_{0}\right)$.

ДОКАЗАТЕЛЬСТВО ШАГА 2.2. Предположим, что любая окрестность $U\left(m_{0}\right)$, гомеоморфная диску, пересекается более чем с одной кривой из $F \cap W^{u}\left(\sigma_{2}\right)$. Тогда существует последовательность точек $m_{k} \in F \cap W^{u}\left(\sigma_{2}\right)$, сходящаяся к точке $m_{0} \in \operatorname{int} F$, такая, что $m_{k}$ лежат на попарно различных компонентах пересечения $F \cap W^{u}\left(\sigma_{2}\right)$. Отсюда и из трансверсальности пересечения $F \cap W^{u}\left(\sigma_{2}\right)$ вытекает, что точки $m_{k}$ изолированы в топологии неустойчивого многообразия $W^{u}\left(\sigma_{2}\right)$. Поэтому $m_{0} \notin W^{u}\left(\sigma_{2}\right)$, иначе неустойчивое многообразие $W^{u}\left(\sigma_{2}\right)$ было бы самопредельньм и существовали бы гомоклинические точки. Так как $M^{3}-\omega=W^{u}\left(\sigma_{2}\right) \cup W^{u}(\alpha) \cup W^{u}\left(\sigma_{1}\right)$, то либо $m_{0} \in W^{u}(\alpha)$, либо $m_{0} \in W^{u}\left(\sigma_{1}\right)$. Включение $m_{0} \in W^{u}(\alpha)$ невозможно, поскольку неустойчивое многообразие $W^{u}(\alpha)$ открыто и не может содержать точек накопления неустойчивого многообразия $W^{u}\left(\sigma_{2}\right)$. Включение $m_{0} \in W^{u}\left(\sigma_{1}\right)$ также невозможно, поскольку в силу $m_{0} \in \operatorname{int} F^{s} \subset W^{u}\left(\sigma_{1}\right)$, оно влечет наличие гомоклинических точек.

Теперь предположим, что $U\left(m_{0}\right)$ пересекается с одной кривой, скажем $l$, но пересечение $U\left(m_{0}\right) \cap l$ содержит компоненту, гомеоморфную простой дуге, которая не делит $U\left(m_{0}\right)$. Из вьшеприведенного рассуждения и трансверсальности пересечения $F \cap$ $W^{u}\left(\sigma_{2}\right)$ вытекает, что предельное множество кривой $l$ в $U\left(m_{0}\right)$ состоит ровно из одной точки. Снова равенство $M^{3}-\omega=W^{u}\left(\sigma_{2}\right) \cup W^{u}(\alpha) \cup W^{u}\left(\sigma_{1}\right)$ приводит к противоречию, так как предельная точка не может принадлежать ни $W^{u}(\alpha)$, ни $W^{u}\left(\sigma_{1}\right)$. Полученное противоречие завершает доказательство шага 2.2 .

ШАГ 2.3. Семейство дуг из пересечения $F^{s} \cap W^{u}\left(\sigma_{2}\right)$, кониевые точки которых лехат на разных граничных компонентах кольиа $F^{s}$, конечно.

ДокАЗАТЕЛЬСТво ШАГА 2.3. Предположим противное. Тогда имеется точка $m_{0} \in$ int $F^{s}$, которая является топологическим пределом попарно различных кривых из $F^{s} \cap$ $W^{u}\left(\sigma_{2}\right)$. Это противоречит шагу 2.2 .

Обозначим через $d=d_{1}, \ldots, d_{k}$ занумерованные в циклическом порядке дуги из пере- 
сечения $F^{s} \cap W^{u}\left(\sigma_{2}\right)$, конщевые точки которых лежат на разных компонентах кольца $F^{s}$. Пусть $\mathcal{D}=\mathcal{D}_{1}, \ldots, \mathcal{D}_{k}-$ кривые из $W^{s}\left(\sigma_{1}\right) \cap W^{u}\left(\sigma_{2}\right)$, содержащие дуги $d=d_{1}, \ldots, d_{k}$ соответственно. Отметим, что некоторые из кривых $\mathcal{D}_{i}$ могут совпадать.

ШАГ 2.4. Среди кривых $\mathcal{D}_{1}, \ldots, \mathcal{D}_{k}$ существует, по крайней мере, одна незамкнутая.

ДоКАЗАТЕЛЬСТВО ШАГА 2.4. Предположим противное. Согласно шагу 2.2 топологический предел кривых из пересечения $F^{s} \cap W^{u}\left(\sigma_{2}\right)$ содержится в граничных компонентах кольца $F^{s}$. Отсюда и из замкнутости кривых $\mathcal{D}_{1}, \ldots, \mathcal{D}_{k}$ следует, что существует замкнутая кривая, непересекающаяся с кривыми из $W^{s}\left(\sigma_{1}\right) \cap W^{u}\left(\sigma_{2}\right)$ и содержащая на устойчивом многообразии $W^{s}\left(\sigma_{1}\right)$ внутри себя точку $\sigma_{1}$ (мы можем "обойти" дуги $d_{1}, \ldots, d_{k}$ вдоль “полукривых" замкнутых кривых $\mathcal{D}_{1}, \ldots, \mathcal{D}_{k}$, при этом мы также можем "обойти" замкнутые кривые из $F^{s} \cap W^{u}\left(\sigma_{2}\right)$, поскольку последние гомотопны нулю в $F^{s}$ ). Это противоречит шагу 2.1 .

Будем для определенности считать, что кривая $\mathcal{D}=\mathcal{D}_{1}$ незамкнута.

ШАГ 2.5. Каждая незамкнутая кривая из $W^{s}\left(\sigma_{1}\right) \cap W^{u}\left(\sigma_{2}\right)$ пересекает все кольиа вида $f^{i}\left(F^{s}\right)$, по крайней мере, одного из оббединений $\bigcup_{i \geqslant 0} f^{i}\left(F^{s}\right)$ или $\bigcup_{i \leqslant 0} f^{i}\left(F^{s}\right)$.

ДоКАЗАТЕЛЬСТВО ШАГА 2.5. Достаточно доказать утверждение для кривой $\mathcal{D}$. Предположим противное. Тогда $\mathcal{D}$ лежит строго внутри конечного объединения $\bigcup_{i=i_{1}}^{i=i_{2}} f^{i}\left(F^{s}\right)$. Из незамкнутости $\mathcal{D}$ вытекает, что внутри этого объединения имеется точка $m_{0}$ такая, что либо любая ее окрестность $U\left(m_{0}\right)$ содержит счетное множество компонент пересечения $U\left(m_{0}\right) \cap \mathcal{D}$, либо $m_{0}$ является единственной предельной точкой одной из полукривых кривой $\mathcal{D}$. Это противоречит шагу 2.2 .

ШАГ 2.6. Каждая незамкнутая кривая из пересечения $W^{s}\left(\sigma_{1}\right) \cap W^{u}\left(\sigma_{2}\right)$ инвариантна относительно некоторой итерации диффеоморфизма $f$.

ДоКАЗАТЕЛЬСТво ШАГА 2.6. Достаточно доказать утверждение для $\mathcal{D}$. Будем для определенности считать, что $\mathcal{D}$ пересекает все кольца из объединения $\bigcup_{i \geqslant 0} f^{i}\left(F^{s}\right)$. Предположим, что $\mathcal{D}$ не инвариантна относительно $f^{i}$ для любого $i \geqslant 0$. Согласно шагу 2.5 для любого $i \geqslant 0$ существует дуга $A_{i}$ кривой $\mathcal{D}$, лежащая в кольце $f^{i}\left(F^{s}\right)$ и имеющая конщевые точки на разных граничных компонентах $f^{i}\left(C_{1}\right)$ и $f^{i}\left(C_{2}\right)$ этого кольца. Так как $\mathcal{D}$ не инвариантна относительно $f^{i}$, дуги $f^{-1}\left(A_{i}\right)$ образуют семейство попарно непересекающихся дуг в кольце $F^{s}$, концевые точки которых лежат на разных граничных компонентах кольца $F^{s}$. Это противоречит шагу 2.3 .

ШАГ 2.7. Кажсдая незамкнутая кривая из $W^{s}\left(\sigma_{1}\right) \cap W^{u}\left(\sigma_{2}\right)$ явяяется кривой без самопересечений с концевыми точками $\sigma_{1}, \sigma_{2}$.

ДоКАЗАТЕЛЬСТВО ШАГА 2.7. Достаточно рассмотреть кривую $\mathcal{D}$. Не уменьшая общности, можно считать, что $\mathcal{D}$ инвариантна относительно $f$. Дуга $d \subset \mathcal{D}$ пересекает фундаментальное кольцо $F^{s}$ в разных окружностях, ограничивающих $F^{s}$. Так как $\bigcup_{i \in \mathbb{Z}} f^{i}\left(F^{s}\right)=W^{s}\left(\sigma_{1}\right)-\sigma_{1}$, то утверждение вытекает из шага 2.6.

ШАГ 2.8. Каждая незамкнутая кривая из пересечения $W^{s}\left(\sigma_{1}\right) \cap W^{u}\left(\sigma_{2}\right)$ после добавления концевых точек $\sigma_{1}$ и $\sigma_{2}$ превращается в непрерывный путь, соединяющ,ий точки $\sigma_{1}, \sigma_{2}$. 
ДоКАЗАТЕЛЬСТВО ШАГА 2.8 достаточно провести для $\mathcal{D}$. В силу шага 2.2 в $F^{s}$ кривая $\mathcal{D}$ не имеет точек накопления. Следовательно, она не имеет точек накопления в любом кольце $f^{i}\left(F^{s}\right)$. Так как в сколь угодно малой окрестности точки $\sigma_{1}$ лежат все кольца $f^{i}\left(F^{s}\right)$, начиная с некоторого момента, $\mathcal{D}$ доопределяется в непрерьвньй путь в точке $\sigma_{1}$. Аналогично доказьвается возможность непрерьвного доопределения в $\sigma_{2}$.

Осталось доказать, что пересечение $W^{s}\left(\sigma_{1}\right) \cap W^{u}\left(\sigma_{2}\right)$ содержит либо нулевое, либо счетное семейство замкнутых кривьх, которые в последнем случае разбиваются в конечное число орбит замкнутых кривых. Действительно, согласно шагу 2.2 внутри фундаментального кольца $F^{s}$ и в окрестностях граничньх кривых кольца $F^{s}$ нет точек накопления замкнутых кривых из $W^{s}\left(\sigma_{1}\right) \cap W^{u}\left(\sigma_{2}\right)$. Отсюда вытекает, что $F^{s}$ пересекает только конечное число замкнутых кривых. Это завершает доказательство теоремы.

Отметим, что кроме $S^{3}$ условию теоремы 2 о накрытии трехмерной сферой удовлетворяют трехмерные сферические пространственные формы, включая линзы (кроме многообразия $S^{2} \times S^{1}$, которое традиционно не включается в список линз). Заметим также, что данное условие существенно: легко построить диффеоморфизм из класса $M S_{1}\left(S^{2} \times S^{1}, 4\right)$ без гетероклинических кривых.

3. Диффеоморфизмы $M S\left(M^{n}, 4\right)$ с седлом коразмерности один. Напомним, что $M S_{n-1}\left(M^{n}, 4\right) \subset M S\left(M^{n}, 4\right)$ означает подкласс диффеоморфизмов, неблуждающее множество которых состоит из трех узлов и одного седла коразмерности один.

ДоКАЗАТЕЛЬСТВО ТЕОРЕМЫ 3. Поскольку утверждение теоремы достаточно доказать для любой итерации $f$, не уменьшая общности, можно считать, что $f: M^{n} \rightarrow M^{n}$ принадлежит $M S\left(M^{n}, 4\right)$, т.е. все периодические точки $f$ неподвижные, и $f$ - сохраняюший ориентацию диффеоморфизм, ограничение которого на инвариантные многообразия неподвижных точек также сохраняет ориентацию. Тогда $f \in M S_{n-1}\left(M^{n}, 4\right)$. Для определенности будем считать, что седло $\sigma$ имеет $(n-1)$-мерную устойчивую сепаратрису $W^{s}(\sigma)$ и две одномерные неустойчивые сепаратрисы $W_{1}^{u}(\sigma), W_{2}^{u}(\sigma)$.

Так как диффеоморфизмы Морса-Смейла не имеют гомоклинических точек, $W^{s}(\sigma)$ может пересекаться только с неустойчивыми многообразиями источников. Пересечение $W^{s}(\sigma)$ с неустойчивым многообразием любого источника открыто в топологии $W^{s}(\sigma)$. Отсюда и из связности множества $W^{s}(\sigma)-\sigma$ вытекает, что $W^{s}(\sigma)-\sigma$ лежит в неустойчивом многообразии ровно одного источника, скажем $\alpha$. Следовательно, $W^{s}(\sigma) \cup$ $\alpha \stackrel{\text { def }}{=} S_{\sigma}^{n-1}$ является вложенной в $M^{n}(n-1)$-мерной сферой с единственно возможной точкой дикости $\alpha$. Однако при $n \geqslant 4$ согласно [3] $S_{\sigma}^{n-1}$ не может иметь изолированную точку дикости. Следовательно, $S_{\sigma}^{n-1}$ цилиндрически вложена в $M^{n}$. Поэтому найдется замкнутая окрестность $K$ сферы $S_{\sigma}^{n-1}$, гомеоморфная замкнутому шаровому слою, такая, что $K$ не будет содержать остальные два узла $\beta_{1}, \beta_{2}$. Покажем, что обе точки $\beta_{i}$ являются стоками.

Предположим противное. Пусть, например, $\beta_{1}-$ сток, а $\beta_{2}$ - источник. Возьмем в малой окрестности $\beta_{2}$ гладкую $(n-1)$-мерную сферу $S_{1}$, ограничивающую замкнутьй $n$-мерньй шар $a_{1}$, которьй содержит точку $\beta_{2}$. Ясно, что все точки из $a_{1}$, кроме $\beta_{2}$, под действием $f$ будут стремиться к $\beta_{1}$. Поэтому найдется такое $k>0$, что $f^{k}\left(S_{1}\right)$ будет принадлежать малой окрестности $U$ точки $\beta_{1}$. Сфера $f^{k}\left(S_{1}\right)$ будет ограничивать в $U$ замкнутый шар $a_{2}$, отличньй от шара $f^{k}\left(a_{1}\right)$. Следовательно, множество $f^{k}\left(a_{1}\right) \cup a_{2}$ будет покрывать все многообразие $M^{n}$, не пересекаясь с точкой $\alpha$, что невозможно. 
Теперь в малой окрестности точки $\beta_{i}$ возьмем $(n-1)$-мерную гладкую сферу $Q_{i}$, ограничивающую $n$-мерньй шар $b_{i}$, которьй содержит $\beta_{i}$ и не имеет общих точек с $K$. Так как обе точки $\beta_{i}$ являются стоками, найдется такое число $k<0$, что $f^{k}\left(Q_{i}\right) \subset$ $K$. Если предположить, что $f^{k}\left(Q_{i}\right)$ ограничивает в $K$ замкнутый шар, скажем $c_{i}$, то множество $f^{k}\left(Q_{i}\right) \cup c_{i}$ совпадало бы с $M^{n}$, что невозможно, поскольку не содержит $S_{\sigma}^{n-1}$. Следовательно, $f^{k}\left(Q_{i}\right)$ не ограничивает в $K$ замкнутых шаров, а сферы $f^{k}\left(Q_{i}\right)$, $S_{\sigma}^{n-1}$ ограничивают в $K$ замкнутый $n$-мерньй шаровой слой $K_{i}$. Тогда $d_{i}=f^{k}\left(b_{i}\right) \cup$ $K_{i}$ будет $n$-мерным замкнутым шаром. Поэтому $M^{n}=d_{1} \cup d_{2}$ и, следовательно, $M^{n}$ является $n$-мерной сферой $S^{n}$.

Так как $M^{n}=S^{n}$, то $S_{\sigma}^{n-1}$ разбивает $S^{n}$ на два $n$-мерных шара $B_{1}^{n}, B_{2}^{n}$. Ясно, что сепаратрисы $W_{1}^{u}(\sigma), W_{2}^{u}(\sigma)$ лежат в разных шарах $B_{1}^{n}, B_{2}^{n}$. Для определенности положим $W_{i}^{u}(\sigma) \subset B_{i}^{n}(i=1,2)$. Отсюда вытекает, что в $B_{1}^{n}$ и $B_{2}^{n}$ лежат стоки $\omega_{1}$ и $\omega_{2}$ соответственно, к которым стремятся сепаратрисы $W_{1}^{u}(\sigma)$ и $W_{2}^{u}(\sigma)$. Это доказывает теорему.

Для исследования вопроса о сопряженности диффеоморфизмов $M S_{n-1}\left(S^{n}, 4\right) \subset$ $M S\left(S^{n}, 4\right)$ решающей является следующая лемма о тривиальности вложения одномерных сепаратрис градиентноподобного диффеоморфизма многообразия $M^{n}$ при $n \geqslant 4$.

Лемма 3.1. Пусть $f: M^{n} \rightarrow M^{n}-$ диффеоморфизм Морса-Смейла замкнутого многообразия $M^{n}, n \geqslant 4$, и псть $s_{0}$ - седловая периодическая точка диффеоморфизма $f$ такая, что $\operatorname{dim} W^{s}\left(s_{0}\right)=n-1 u \operatorname{dim} W^{u}\left(s_{0}\right)=1$. Предположим, что имеется сепаратриса $W_{1}^{u}\left(s_{0}\right) \subset W^{u}\left(s_{0}\right)-s_{0}$ точки $s_{0}$ и периодическая стоковая точка $S_{1}$ такие, что clos $W_{1}^{u}\left(s_{0}\right)=s_{0} \cup W_{1}^{u}\left(s_{0}\right) \cup S_{1}$. Тогда

1) $\operatorname{clos} W_{1}^{u}\left(s_{0}\right)=s_{0} \cup W^{u}\left(s_{0}\right) \cup S_{1}$ является одномерным ручным полиәдром;

2) существует гладко вложсенная $(n-1)$-сфера $S^{n-1} \subset W^{s}\left(S_{1}\right)$, окружающая $n$-иар $B^{n} \subset W^{s}\left(S_{1}\right)$ с точкой $S_{1}$ внутри, такая, что $W_{1}^{u}\left(s_{0}\right)$ пересекается

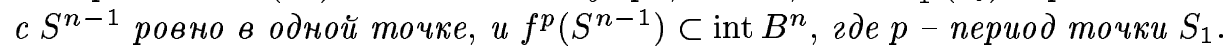

ДокАЗАТЕЛЬСТво. Очевидно, объединение

$$
S_{1} \cup W_{1}^{u}\left(s_{0}\right) \cup s_{0} \stackrel{\text { def }}{=} P_{1}\left(s_{0}\right)
$$

является компактной дугой, гомеоморфной отрезку [0, 1], т.е. одномерным вложенным полиэдром. Покажем, что этот полиэдр ручной. Действительно, так как сепаратриса $W_{1}^{u}\left(s_{0}\right)$ является гладко вложенной кривой и, следовательно, не имеет точек дикости, одномерный полиэдр $P\left(s_{0}\right)$ может иметь не более одной точки дикости, $S_{1}$. Но в силу [14] одномерньй полиэдр в пространстве размерности $n \geqslant 4$ либо имеет совершенное (в частности, бесконечное) множество точек дикости, либо является ручным. Следовательно, одномерный полиэдр $P\left(s_{0}\right)$ ручной.

Построим теперь $(n-1)$-сферу, удовлетворяющую пункту 2$)$. Для простоты предполагаем, что обе точки $s_{0}, S_{1}$ неподвижные относительно $f$ (в противном случаеперейдем к некоторой итерации $f$ ). Так как $S_{1}$ является гиперболической притягивающей точкой, существует гладко вложенная $(n-1)$-сфера $S_{1}^{n-1} \subset W^{s}\left(S_{1}\right)$, окружающая $n$-шар $B_{1}^{n} \subset W^{s}\left(S_{1}\right)$ с точкой $S_{1}$ внутри, такая, что $f\left(S_{1}^{n-1}\right) \subset \operatorname{int} B_{1}^{n}$. Пошевелив, если необходимо, сферу $S_{1}^{n-1}$, можно добиться того, чтобы $S_{1}^{n-1}$ трансверсально пересекалась c $W_{1}^{u}\left(s_{0}\right)$. 
Очевидно, $f\left(S_{1}^{n-1}\right) \subset W^{s}\left(S_{1}\right)$ и $f^{-1}\left(S_{1}^{n-1}\right) \subset W^{s}\left(S_{1}\right)$. Так как $W^{s}\left(S_{1}\right) \cong \mathbb{R}^{n}$, гладко вложенные $(n-1)$-сферы $f\left(S_{1}^{n-1}\right)$ и $f^{-1}\left(S_{1}^{n-1}\right)$ ограничивают в $W^{s}\left(S_{1}\right)$ замкнутое множество $A_{0}=\operatorname{clos} f^{-1}\left(B_{1}^{n}\right)-\operatorname{int} f\left(B_{1}^{n}\right)$, гомеоморфное шаровому слою $\Sigma^{n-1} \times[0 ; 1]$, где $\Sigma^{n-1}-$ стандартная $(n-1)$-сфера. Ниже, для краткости, шаровой слой мы иногда будем назьвать кольцом. Аналогично

$$
A_{1}=\operatorname{clos} f^{-1}\left(B_{1}^{n}\right)-\operatorname{int} B_{1}^{n}, \quad A_{2}=\operatorname{clos} B_{1}^{n}-\operatorname{int} f\left(B_{1}^{n}\right)
$$

также гомеоморфны кольцу $\Sigma^{n-1} \times[0 ; 1]$. Отметим, что каждое из колец $A_{1}, A_{2}$ является фундаментальным, т.е. итерации любого кольца образуют область притяжения стока $S_{1}$ (кроме точки $S_{1}$ ), и внутри каждого кольца нет различных точек одной орбиты. (Такое кольцо иногда называют порождающим.)

В силу инвариантности $W_{1}^{u}\left(s_{0}\right)$ относительно $f$ все сферы $S_{1}^{n-1}, f\left(S_{1}^{n-1}\right), f^{-1}\left(S_{1}^{n-1}\right)$ трансверсальны сепаратрисе $W_{1}^{u}\left(s_{0}\right)$. Ориентируем $W_{1}^{u}\left(s_{0}\right)$ от $s_{0}$ к $S_{1}$. Связные компоненты пересечения $A_{i} \cap W_{1}^{u}\left(s_{0}\right)(i=0,1,2)$ суть дуги с концевьми точками, содержащимися в одной из сфер $S_{1}^{n-1}, f\left(S_{1}^{n-1}\right), f^{-1}\left(S_{1}^{n-1}\right)$. В силу трансверсальности пересечения, условия 1$)$ и равенства $\operatorname{clos} W_{1}^{u}\left(s_{0}\right)=s_{0} \cup W_{1}^{u}\left(s_{0}\right) \cup S_{1}$ число дуг конечно. Если конщевые точки любой дуги $A_{i} \cap W_{1}^{u}\left(s_{0}\right)$ лежат на разных сферах, то $S_{1}^{n-1}$ удовлетворяет требованию теоремы, поскольку кольца $A_{1}, A_{2}$ порождающие и, следовательно, каждое из них $W_{1}^{u}\left(s_{0}\right)$ пересекает ровно по одной дуге. Поэтому в дальнейшем будем предполагать, что в кольцах $A_{1}, A_{2}$ дуги с концевыми точками на одной сфере существуют. Из геометрических соображений следует, что тогда имеются дуги с обоими конщевыми точками на $S_{1}^{n-1}$, которые входят и выходят из каждого кольца $A_{1}, A_{2}$.

Рассмотрим дуги $a_{1}, \ldots, a_{k_{1}} \subset A_{1} \cap W_{1}^{u}\left(s_{0}\right)$ и дуги $a_{k_{1}+1}, \ldots, a_{k_{2}} \subset A_{2} \cap W_{1}^{u}\left(s_{0}\right)$, все конщевые точки которых принадлежат сфере $S_{1}^{n-1}$. Положим

$$
\mathcal{D}_{1}=\bigcup_{i=1}^{k_{1}} a_{i}, \quad \mathcal{D}_{2}=\bigcup_{i=k_{1}+1}^{k_{2}} a_{i} .
$$

Отметим, что в силу трансверсальности дуги, входящие в $A_{1}$ (равно как и в $A_{2}$ ), имеют различные концевые точки. Для каждого $1 \leqslant i \leqslant k_{1}$ соединим концевые точки дуги $a_{i}$ путем $e_{i} \subset S_{1}^{n-1}$ без самопересечений так, что пересечение $e_{i} \cap W_{1}^{u}\left(s_{0}\right)$ состоит только из концевых точек $e_{i}$. Более того, так как $n \geqslant 4$, то, не уменьшая общности, можно считать, что $e_{i}$ попарно не пересекаются (см. рис. 5). Тогда объединение $c_{i}=a_{i} \cup e_{i}$ является простой замкнутой кривой, $i=1, \ldots, k_{1}$. Так как $n \geqslant 4, c_{i}$ ограничивает замкнутый полиэдральный диск без самопересечений [15], который мы обозначим через $D_{i}$, и, более того, мы можем считать, что $D_{i}$ пересекает сепаратрису $W_{1}^{u}\left(s_{0}\right)$ только по дуге $a_{i}$. Снова в силу неравенства $n \geqslant 4$ и двумерности всех дисков $D_{1}, \ldots, D_{k_{1}}$ можно их пошевелить, не меняя вблизи кривых $c_{i}$, так, чтобы они попарно не пересекались и каждьй диск не пересекался с дугами из $\mathcal{D}_{2} \cup f^{-1}\left(\mathcal{D}_{2}\right)[15]$.

Покажем, что можно заменить диск $D_{1}$ на полиэдральный диск без самопересечений с той же гранищей, который по-прежнему не будет пересекаться с остальными дисками $D_{2}, \ldots, D_{k_{1}}$, с дугами из $\mathcal{D}_{2} \cup f^{-1}\left(\mathcal{D}_{2}\right)$, и которьй вдобавок будет пересекаться с $S_{1}^{n-1}$ только по кривой $e_{1}$. Пошевелим $D_{1}$ так, чтобы $D_{1}$ находился в общем положении относительно сфер $S_{1}^{n-1}, f\left(S_{1}^{n-1}\right)$ и $f^{-1}\left(S_{1}^{n-1}\right)$ и не пересекался с $D_{2}, \ldots, D_{k_{1}}$, $\mathcal{D}_{2} \cup f^{-1}\left(\mathcal{D}_{2}\right)$. Тогда каждая компонента пересечения

$$
\text { int } D_{1} \cap\left(S_{1}^{n-1} \cup f\left(S_{1}^{n-1}\right) \cup f^{-1}\left(S_{1}^{n-1}\right)\right)
$$




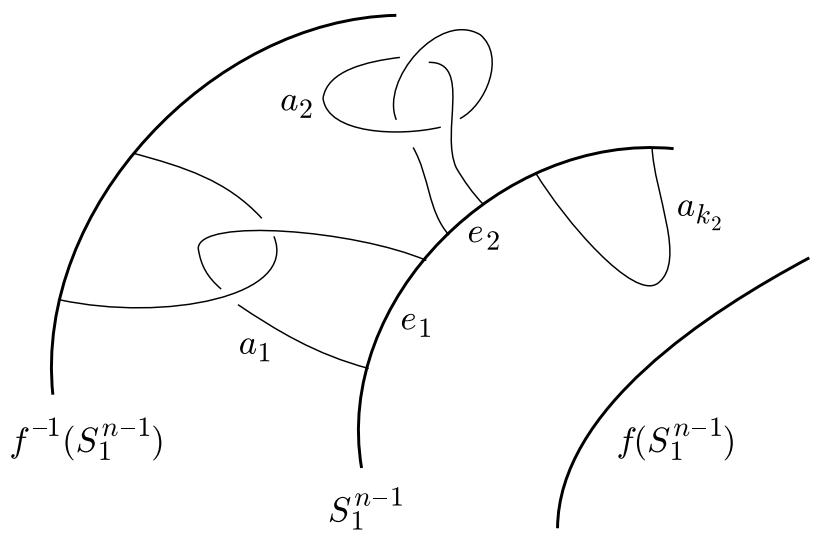

Рис. 5. Дуги $a_{i}$ и $e_{i}$.

является либо простой замкнутой кривой, либо открытой дугой (причем в последнем случае концевые точки, возможно совпадающие, принадлежат $e_{1}$ ). Пусть $d$ - простая замкнутая кривая, являющаяся компонентой пересечения (1), которая не содержит внутри себя на диске $D_{1}$ других компонент из этого пересечения. Для определенности будем считать, что $d \subset S_{1}^{n-1}$ (в случае $d \subset f\left(S_{1}^{n-1}\right)$ или $d \subset f^{-1}\left(S_{1}^{n-1}\right)$ рассуждения полностью аналогичные). Очевидно, сфера $S_{1}^{n-1}$ разбивает $M^{n}$ на две компоненты, одна из которых является шаром $B_{1}^{n}$. Обозначим через $b_{1} \subset D_{1}$ диск, ограниченньй кривой $d$, и через $K_{1}, K_{2}$ - компоненты множества $M^{n}-S_{1}^{n-1}$ с $b_{1} \subset \operatorname{clos} K_{1}$. Возьмем точку $x_{0} \in K_{2}$, достаточно близкую к $S_{1}^{n-1}$, так, чтобы конус $k$ с основанием $d$ и вершиной $x_{0}$ лежал в $\operatorname{clos} K_{1}$. Так как $n \geqslant 4$, пошевелив $k$, если необходимо, можно добиться того, чтобы $k$ не пересекался с $D_{2}, \ldots, D_{k_{1}}, \mathcal{D}_{2} \cup f^{-1}\left(\mathcal{D}_{2}\right)$. Заменим $D_{1}$ на диск $\left(D_{1}-b_{1}\right) \cup k$. Этот диск продеформируем в $K_{2}$ так, чтобы у полученного диска $D_{1}^{\prime}$ не возникло новых пересечений с $S_{1}^{n-1} \cup f\left(S_{1}^{n-1}\right) \cup f^{-1}\left(S_{1}^{n-1}\right)$. Тогда $D_{1}^{\prime}$ имеет на одну кривую меньше пересечений с $S_{1}^{n-1} \cup f\left(S_{1}^{n-1}\right) \cup f^{-1}\left(S_{1}^{n-1}\right)$, чем $D_{1}$. Полностью аналогично “убираются" открытые дуги из пересечений (1). В итоге мы получим требуемьй диск (обозначим его снова через $\left.D_{1}\right)$, которьй не пересекается с остальньми дисками $D_{2}, \ldots, D_{k_{1}}$, $\mathcal{D}_{2} \cup f^{-1}\left(\mathcal{D}_{2}\right)$, и которьй пересекается с $S_{1}^{n-1}$ только по кривой $e_{1}$, но не пересекается c $f\left(S_{1}^{n-1}\right) \cup f^{-1}\left(S_{1}^{n-1}\right)$.

Поскольку дуга $a_{1}$ входит в кольцо $A_{1}$, и во время преобразований гранища $D_{1}$ не менялась, то полученный диск $D_{1}$ лежит в $A_{1}$.

Осуществив аналогичную процедуру с остальными дисками, получим попарно непересекающиеся диски $D_{1}, \ldots, D_{k_{1}}$ (обозначим их теми же буквами), каждьй из которых не персекается с $\mathcal{D}_{2} \cup f^{-1}\left(\mathcal{D}_{2}\right)$, и при этом $D_{i}$ пересекается с $S_{1}^{n-1}$ только по кривой $e_{i}, 1 \leqslant i \leqslant k_{1}$. Более того, $D_{i}$ пересекает сепаратрису $W_{1}^{u}\left(s_{0}\right)$ только по дуге $a_{i}$. По построению каждый $D_{i}$ лежит в $A_{1}$ и является ручным полиэдром без самопересечений.

В силу компактности дисков $D_{1}, \ldots, D_{k_{1}}$ существуют попарно не пересекающиеся окрестности $U\left(D_{1}\right), \ldots, U\left(D_{k_{1}}\right)$ этих дисков соответственно, каждая из которых является гладко вложенньм открытым $n$-шаром с гладкой границей $\Sigma_{i}=\partial U\left(D_{i}\right)$, гомеоморфной $(n-1)$-сфере. Уменьшив, если необходимо, каждую окрестность, будем считать, что

$$
U\left(D_{i}\right) \cap\left(f^{-1}\left(S_{1}^{n-1}\right) \cup f\left(S_{1}^{n-1}\right)\right)=\varnothing, \quad U\left(D_{i}\right) \cap f^{-1}\left(\mathcal{D}_{2}\right)=\varnothing, \quad 1 \leqslant i \leqslant k_{1} .
$$


Более того, так как $D_{i}$ является ручньм полиэдром без самопересечений, можно также считать, что пересечение $U\left(D_{i}\right) \cap S_{1}^{n-1}$ состоит из $(n-1)$-шара $B_{i}^{n-1} \subset S_{1}^{n-1}$, который содержит $e_{i}$ и делит $U\left(D_{i}\right)$ на два открытых $n$-шара, $B_{1} \subset A_{1}$ и $B_{2} \subset A_{2}$. Не уменьшая общности, можно считать, что сфера $\Sigma_{i}=\partial U\left(D_{i}\right)$ пересекает $S_{1}^{n-1}$ трансверсально. Тогда $\Sigma_{i}$ разбивается границей $S_{i}^{n-2}=\partial B_{i}^{n-1}$ на два замкнутых $(n-1)$-шара $D_{1, i}^{n-1} \subset$ $\Sigma_{i} \cap A_{1}$ и $D_{2, i}^{n-1} \subset \Sigma_{i} \cap A_{2}$. Положим

$$
S_{*}^{n-1}=\left(S_{1}^{n-1}-\bigcup_{i=1}^{k_{1}} B_{i}^{n-1}\right) \bigcup_{i=1}^{k_{1}} D_{1, i}^{n-1}
$$

В силу свойств окрестностей $U\left(D_{i}\right), S_{*}^{n-1}$ является цилиндрически вложенной $(n-1)$-сферой со следуюшими свойствами:

1) $S_{*}^{n-1} \subset A_{1}, S_{*}^{n-1} \cap f^{-1}\left(S_{1}^{n-1}\right)=\varnothing$;

2) $S_{*}^{n-1} \cap\left(\mathcal{D}_{1} \cup f^{-1}\left(\mathcal{D}_{2}\right)\right)=\varnothing$.

Так как кольцо $A_{1}$ является порождаюшим, в силу свойства 1)

$$
S_{*}^{n-1} \cap f^{-1}\left(S_{*}^{n-1}\right)=\varnothing, \quad S_{*}^{n-1} \cap f\left(S_{*}^{n-1}\right)=\varnothing .
$$

В силу трансверсальности пересечения сфер $\Sigma_{i}, S_{1}^{n-1}$ и трансверсальности пересечения $W^{u}\left(s_{0}\right) \cap S_{1}^{n-1}$ можно сгладить $S_{*}^{n-1}$ вблизи $\Sigma_{i} \cap S_{1}^{n-1}$ так, чтобы полученная гладко вложенная сфера $S^{n-1}$ удовлетворяла следующим условиям:

а) $S^{n-1} \subset A_{1}, S^{n-1} \cap f^{-1}\left(S^{n-1}\right)=\varnothing$;

б) $S^{n-1} \cap\left(\mathcal{D}_{1} \cup f^{-1}\left(\mathcal{D}_{2}\right)\right)=\varnothing$;

в) пересечение $W^{u}\left(s_{0}\right) \cap S^{n-1}$ трансверсально.

По построению все окрестности $U\left(D_{i}\right)$ лежат в $W^{s}\left(S_{1}\right)$. Поэтому $S^{n-1}, f\left(S^{n-1}\right)$, $f^{-1}\left(S^{n-1}\right) \subset W^{s}\left(S_{1}\right)$. В силу свойства а) $(n-1)$-мерные сферы $S^{n-1}, f^{-1}\left(S^{n-1}\right)$ ограничивают замкнутое $n$-мерное кольцо $A^{n} \subset W^{s}\left(S_{1}\right)$, поскольку $W^{s}\left(S_{1}\right) \cong \mathbb{R}^{n}$. Следовательно, $A^{n}$ является порождающим, т.е.

$$
\bigcup_{j \in \mathbb{Z}} f^{j}\left(A^{n}\right)=W^{s}\left(S_{1}\right)-S_{1}, \quad \text { int } f^{i}\left(A^{n}\right) \cap \operatorname{int} f^{l}\left(A^{n}\right)=\varnothing, \quad \text { если } i \neq l .
$$

Отсюда и из свойства б) вытекает, что нет дуг сепаратрисы $W^{s}\left(S_{1}\right)$, которые входят в кольцо $A^{n}$ через одну из сфер $S^{n-1}$ или $f^{-1}\left(S^{n-1}\right)$ и возвращаются, не выходя из $A^{n}$, на ту же сферу. Это влечет, что $A^{n}$ пересекается с $W^{s}\left(S_{1}\right)$ ровно по одной дуге, концевые точки которой лежат на разных компонентах гранищы $\partial A^{n}$.

По построению $(n-1)$-сфера $S^{n-1} \subset M^{n}$ окружает $n$-шар $B^{n}$ с точкой $S_{1}$. Это завершает доказательство леммы.

Теперь докажем теорему 4, которая показывает, что индекс Морса (который принимает только два значения: либо 1 , либо $n-1)$ седла является полньм инвариантом сопряженности диффеоморфизмов из класса $M S_{n-1}\left(S^{n}, 4\right)$. Обозначим через $\sigma_{f}$ седло коразмерности один диффеоморфизма $f \in M S_{n-1}\left(S^{n}, 4\right)$.

ДоКАЗАТЕЛЬСТВо ТЕОРЕМЫ 4. Очевидно, если $f, g \in M S_{n-1}\left(S^{n}, 4\right)$ сопряжены, то седла $\sigma_{f}, \sigma_{g}$ имеют одинаковьй индекс Морса. Докажем обратное утверждение. Для 
определенности будем считать, что индекс Морса седел $\sigma_{f}, \sigma_{g}$ равен 1 (в противном случае перейдем к обратным диффеоморфизмам). Тогда седло $\sigma_{f}$ имеет $(n-1)$-мерное устойчивое многообразие $W^{s}\left(\sigma_{f}\right)$ и две одномерные неустойчивые сепаратрисы $W_{1}^{u}\left(\sigma_{f}\right)$, $W_{2}^{u}\left(\sigma_{f}\right)$. Согласно теореме 3 сепаратрисы $W_{1}^{u}\left(\sigma_{f}\right), W_{2}^{u}\left(\sigma_{f}\right)$ содержат в своем замыкании различные стоки $\omega_{1, f}$ и $\omega_{2, f}$ соответственно. В силу леммы 3.1 существует гладко вложенная $(n-1)$-сфера $S_{i, f} \subset W^{s}\left(\omega_{i, f}\right)$, окружаюшая $n$-шар $B_{i, f} \subset W^{s}\left(\omega_{i, f}\right)$ с точкой $\omega_{i, f}$ внутри, такая, что $W_{i}^{u}\left(\sigma_{f}\right)$ пересекается с $S_{i, f}$ ровно в одной точке $a_{i, f}$, и $f\left(S_{i, f}\right) \subset \operatorname{int} B_{i, f}, i=1,2$. Тогда $W_{i}^{u}\left(\sigma_{f}\right)$ пересекается с $f\left(S_{i, f}\right)$ также ровно в одной точке $b_{i, f}=f\left(a_{i, f}\right)$. Так как сфферы $S_{i, f}, f\left(S_{i, f}\right) \subset \operatorname{int} B_{i, f}$ гладко вложены, они ограничивают замкнутьй шаровой слой, которьй мы обозначим через $A_{i, f}$. Не уменьшая общности, можно считать, что сеператриса $W_{i}^{u}\left(\sigma_{f}\right)$ пересекает $S_{i, f}$ трансверсально. Поэтому $W_{i}^{u}\left(\sigma_{f}\right)$ пересекает сферу $f\left(S_{i, f}\right)$ также трансверсально. Следовательно, существует замкнутое множество $U\left(b_{1, f}, b_{2, f}\right)$, содержащее дугу $\left[b_{1, f} b_{2, f}\right]^{u} \subset W^{u}\left(\sigma_{f}\right)$, со следуюшими свойствами (внутренность $U\left(b_{1}, f, b_{2}, f\right)$ можно рассматривать как трубчатую окрестность чуть уменьшенной дуги $\left[b_{1, f} b_{2, f}\right]^{u}$, см. рис. 6$)$ :

1) $U\left(b_{1, f}, b_{2, f}\right) \cap A_{i, f} \stackrel{\text { def }}{=} d_{i, f}$ является $n$-шаром, ограниченным $(n-1)$-мерными шарами $U\left(b_{1, f}, b_{2, f}\right) \cap S_{i, f}, U\left(b_{1, f}, b_{2, f}\right) \cap f\left(S_{i, f}\right)$ и шаровьм кольцом $\partial U\left(b_{1}, f, b_{2, f}\right) \cap$ $A_{i, f}$

2) $f\left(U\left(b_{1, f}, b_{2, f}\right)\right) \cap A_{i, f} \subset U\left(b_{1, f}, b_{2, f}\right)$;

3) $U\left(\sigma_{f}\right) \stackrel{\text { def }}{=} \bigcup_{j \in \mathbb{Z}} f^{j}\left(U\left(b_{1, f}, b_{2, f}\right)\right) \cup \omega_{1, f} \cup \omega_{2, f}$ содержит неустойчивое и устойчивое многообразия $W^{u}\left(\sigma_{f}\right), W^{s}\left(\sigma_{f}\right)$.

Аналогичные обозначения и объекты введем для диффеоморфизма $g$.

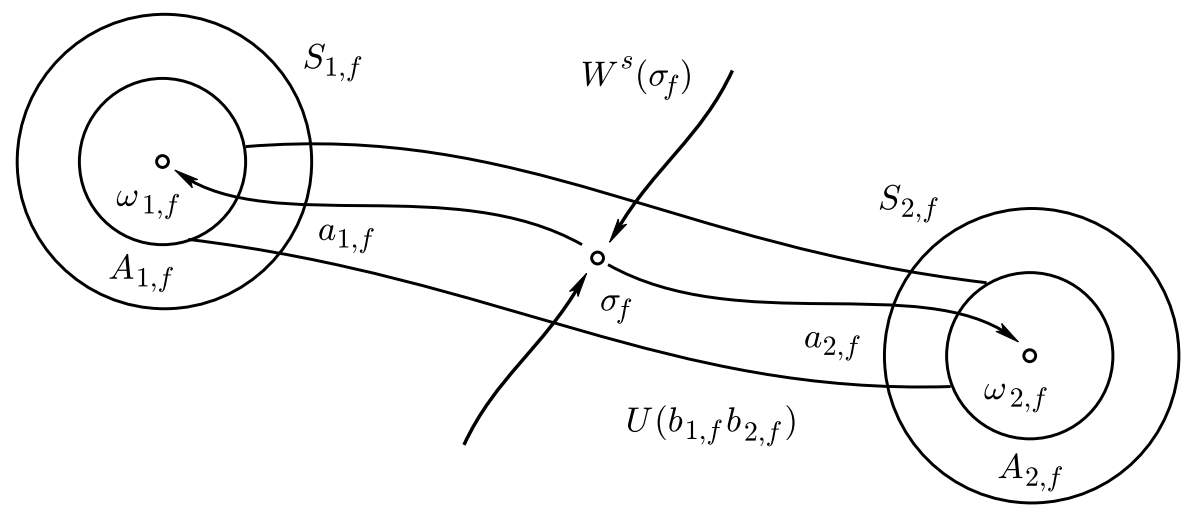

Рис. 6. Множество $U\left(b_{1, f}, b_{2, f}\right)$.

Так как седла $\sigma_{f}, \sigma_{g}$ имеют одинаковьй индекс Морса, в силу теоремы ГробманаХартмана [8] диффеоморфизмы $f$ и $g$ сопряжены в окрестности седел $\sigma_{f}, \sigma_{g}$. Для достаточно “малого" $U\left(b_{1, f}, b_{2, f}\right)$ можно считать, что $f$ и $g$ сопряжены в $U\left(b_{1, f}, b_{2, f}\right)$. Отсюда вытекает, что существует гомеоморфизм $h_{1}: U\left(\sigma_{f}\right) \rightarrow U\left(\sigma_{g}\right)$ такой, что

$$
\left.h_{1} \circ f\right|_{U\left(\sigma_{f}\right)}=\left.g \circ h_{1}\right|_{U\left(\sigma_{f}\right)} .
$$

Более того, в силу трансверсальности пересечений $W^{u}\left(\sigma_{f}\right) \cap S_{i, f}, W^{u}\left(\sigma_{g}\right) \cap S_{i, g}(i=$ $1,2)$ и достаточной свободы построения сфер $S_{i, f}, S_{i, g}$ можно построить гомеоморфизм 
$h_{1}$ таким образом, чтобы вьполнялись следующие соотношения:

$$
h_{1}\left(U\left(b_{1, f}, b_{2, f}\right) \cap S_{i, f}\right)=U\left(b_{1, g}, b_{2, g}\right) \cap S_{i, g}, \quad i=1,2 .
$$

Дополнения сффер $S_{i, f}, f\left(S_{i, f}\right)$ к $(n-1)$-мерньм шарам $U\left(b_{1, f}, b_{2, f}\right) \cap S_{i, f}, U\left(b_{1, f}, b_{2, f}\right) \cap$ $f\left(S_{i, f}\right)$ соответственно являются $(n-1)$-мерными шарами. Продолжим $h_{1}$ на $S_{i, f} \cup$ $f\left(S_{i, f}\right)$ до гомеоморфизма $S_{i, f} \cup f\left(S_{i, f}\right) \rightarrow S_{i, g} \cup f\left(S_{i, g}\right)$, который обозначим той же буквой $h_{1}$, таким образом, чтобы вьполнялось соотношение

$$
\left.h_{1} \circ f\right|_{S_{i, f}}=\left.g \circ h_{1}\right|_{S_{i, g}} .
$$

Согласно построению замькание $\cos \left(A_{i, f}-d_{i, f}\right)$ является $n$-шаром, на гранище которого задан $h_{1}$. Продолжим $h_{1}$ на весь шар $\operatorname{clos}\left(A_{i, f}-d_{i, f}\right)$ произвольным образом до гомеоморфизма $\operatorname{clos}\left(A_{i, f}-d_{i, f}\right) \rightarrow \operatorname{clos}\left(A_{i, g}-d_{i, g}\right)$, которьй мы обозначим через $h_{2}$. Поскольку кольца $A_{i, f}, i=1,2$, являются порождаюшими для ограничения $f$ на $W^{s}\left(\omega_{i, f}\right), h_{2}$ естественньм образом продолжается до гомеоморфизма

$$
h_{2}: W^{s}\left(\omega_{1, f}\right) \cup W^{s}\left(\omega_{2, f}\right) \rightarrow W^{s}\left(\omega_{1, g}\right) \cup W^{s}\left(\omega_{2, g}\right)
$$

такого, что

$$
h_{2}\left(\omega_{i, f}\right)=\omega_{i, g},\left.\quad h_{2} \circ f\right|_{W^{s}\left(\omega_{1, f}\right) \cup W^{s}\left(\omega_{2, f}\right)}=\left.g \circ h_{2}\right|_{W^{s}\left(\omega_{1, f}\right) \cup W^{s}\left(\omega_{2, f}\right)} .
$$

Так как $h_{1}$ и $h_{2}$ совпадают на границе шара $d_{i, f}$, они образуют гомеоморфизм $h$, которьй продолжается до гомеоморфизма всей сферы $S^{n}$, если положить $h\left(\alpha_{f}\right)=\alpha_{g}$. Нетрудно видеть, что для $h$ вьполняется равенство $g \circ h=h \circ f$, что доказывает теореMy.

Авторы благодарят Д. В. Аносова и Х. Бонатти за плодотворные обсуждения. Авторы благодарят А. Ю. Жирова, прочитавшего внимательно рукопись и указавшего на ряд неточностей.

\section{СПИСОК ЦИТИРОВАННОЙ ЛИТЕРАТУРЫ}

[1] Smale S. Differentiable dynamical systems // Bull. Amer. Math. Soc. 1967. V. 73. №1. P. 741-817.

[2] Безденежных А. Н., Гринес В. З. Динамические свойства и топологическая классификация градиентноподобных диффеоморфизмов на двумерных многообразиях I (II) // Методы КТДУ / ред. Е. А. Лентович-Андронова. Горький: ГГУ, 1985. С. 22-38; 1987. С. 24-32. 
[3] Безденежных А. Н., Гринес В. З. Реализация градиентноподобных диффеоморфизмов двумерных многообразий // Дифференц. и интегральные уравнения / ред. Н. Ф. Отроков. Горький: ГГУ, 1985. С. 33-37.

[4] Pixton D. Wild unstable manifolds // Topology. 1977. V. 16. P. 167-172.

[5] Bonatti Ch., Grines V. Z. Knots as topological invariant for gradient-like diffeomorphisms of the sphere $S^{3} / /$ J. of Dyn. and Control Syst. 2000. V. 6. P. 579-602.

[6] Бонатти Х., Гринес В. З., Медведев В. С., Пеку Е. О топологической классификации градиентноподобных диффеоморфизмов без гетероклинических кривых на трехмерных многообразиях // Докл. РАН. 2001. Т. 377. № 2. С. 151-155.

[7] Fox R. H., Artin E. Some wild cells and spheres in three-dimensional space // Ann. of Math. 1948. V. 49. P. 979-990.

[8] Нитецки З. Введение в дифференциальную динамику. М.: Мир, 1975.

[9] Аносов Д. В. Исходные понятия. Элементарная теория // Современные проблемы математики. Динамические системы-1. Т. 1, 1985. С. 156-178, 178-204.

[10] Арансон С. Х., Гринес В. З. Каскады на поверхностях // Современные проблемы математики. Динамические системы-9. Т. 66, 1991. С. 148-187.

[11] Келдыш Л. В. Топологические вложения в евклидово пространство // Тр. МИАН. 1966.

[12] Bonatti Ch., Grines V. Z., Medvedev V., Pecou E. Three-dimensional manifolds admitting Morse-Smale diffeomorphisms without heteroclinic curves // Topology and applications. 2002. V. 117. P. 335-344.

[13] Cantrell J. C. Almost locally flat embeddings of $S^{n-1}$ in $S^{n} / /$ Bull. Amer. Math. Soc. 1963. V. 69. P. 716-718.

[14] Cantrell J. C., Edwards C. H. Almost locally polyhedral curves in Euclidean $n$-space // Trans. Amer. Math. Soc. 1963. V. 107. № 3. P. 451-457.

[15] Gugenheim V. Piecewise linear isotopy and embedding of elements and spheres I // Proc. London Math. Soc. 1953. V. 3. P. 29-53.

(В.З. Гринес) Нижегородская государственная

Поступило сельскохозяйственная академия;

(Е. В. Жужома) Нижегородский государственный технический университет;

12.09.2001

Исправленный вариант

(В. С. Медведев) Институт прикладной математики и кибернетики

22.05 .2002

при Нижегородском государственном университете

им. Н. И. Лобачевского

E-mail:

grines@unn.ac.ru

zhuzhoma@mail.ru

medvedev@unn.ac.ru 